\title{
Sensitivity comparison of localized plasmon resonance structures and prism coupler
}

\author{
Yasin Kaya ${ }^{\mathrm{a}}$, Sencer Ayas ${ }^{\mathrm{b}}$, Ahmet Emin Topal ${ }^{\mathrm{b}}$, Hasan Guner ${ }^{\mathrm{b}}$, Aykutlu Dana ${ }^{\mathrm{b}, *}$ \\ a Department of Electrical and Electronics Engineering, Bilkent University, 06800 Ankara, Turkey \\ ${ }^{\mathrm{b}}$ UNAM Institute of Materials Science and Nanotechnology, Bilkent University, 06800 Ankara, Turkey
}

\section{A R T I C L E I N F O}

\section{Article history:}

Received 18 July 2013

Received in revised form

16 September 2013

Accepted 25 September 2013

Available online 18 October 2013

\section{Keywords:}

Surface plasmon resonance

Biomolecular sensing

Localized plasmon resonance sensors

\begin{abstract}
A B S T R A C T
Plasmon resonances are widely used in biomolecular sensing and continue to be an active research field due to the rich variety of surface and measurement configurations, some of which exhibit down to single molecule level sensitivity. The resonance wavelength shift of the plasmonic structure upon binding of molecules, strongly depends, among other parameters, on how well the field of the resonant mode is confined to the binding site. Here it is shown that, by using properly designed metal-insulatormetal type resonators, improved wavelength response can be achieved with localized surface plasmon resonators (LSPRs) compared to that of the commonly used Kretschmann geometry. Using computational tools we investigate theoretically the refractive index response of several LSPR structures to a $2 \mathrm{~nm}$ thin film of binding molecules. LSPR resonators are shown to feature improved sensitivity over conventional Kretschmann geometry in the wavelength interrogation scheme for such a thin film. Moreover, some of the LSPR modes are quasi-omnidirectional and such angular independence (up to $30^{\circ}$ angle of incidence) allows higher numerical apertures to be used in colorimetric imaging. Results highlight the potential of LSPRs for biomolecular sensing with high sensitivity and high spatial resolution.
\end{abstract}

(C) 2013 Elsevier B.V. All rights reserved.

\section{Introduction}

Resonance excitation and detection of Surface Plasmon Polaritons (SPPs) provide a versatile platform for affinity based refractometric biomolecular sensing [1,2]. Typically, the refractive index profile near a metal surface is modified due to the refractive index contrast between a buffer solution and attached biomolecules and the resonance of the plasmonic modes are shifted frequency, which can be monitored using reflection or transmission measurements. Due to the ease of far field measurement and the simplicity of fabricating surfaces exhibiting plasmonic resonances, surface plasmon resonance (SPR) technique has been extensively used over the last few decades for biomolecular sensing [3-5]. Commonly, prism and grating coupling have been used to excite SPPs on planar or quasi-planar surfaces, and prism coupling is accepted to exhibit higher sensitivity [6]. The advent of nanofabrication tools allowed more and more top-down structures to be employed in plasmonic sensing. The rich physics of single or coupled plasmonic structures allows high performance biomolecular sensing using ordered periodic localized plasmon mode resonances (LSPR) [7-9]. Typically, SPR imaging allows simultaneous detection from multiple individually functionalized spots. As prism coupled SPR has a

\footnotetext{
* Corresponding author. Tel.: +90 3122903502.

E-mail address: aykutlu@unam.bilkent.edu.tr (A. Dana).
}

strong angular dependence of the resonant excitation, a low numerical aperture has to be used, and SPR imaging has been inherently a low spatial resolution technique $[10,11]$. In order to perform plasmon resonance imaging with high spatial resolution, it is desirable to have plasmonic surfaces that are not sensitive to angle of excitation (omnidirectionality), while maintaining a high refractive index sensitivity, thereby allowing sensing of monolayer biomolecular coatings. Periodic localized plasmon structures can be designed with optical band structures which exhibit quasi-omnidirectional response [12].

In this article, we explore theoretically the refractive index sensitivity of several plasmonic designs. Typically, a simple figureof-merit (FOM), defined as the ratio of sensitivity (shift of the resonance wavelength in $\mathrm{nm}$ per change in the bulk refractive index of the sensing medium) to the full-width-at-half-maximum (FWHM) of the resonant line shape, is used to compare the performance $[13,14]$. We note that, in biomolecular sensing applications, the performance depends not on bulk refractive index change but on molecular binding. Therefore, instead of using the bulk refractive index change for the comparison of sensitivity of structures with that of a prism, a thin ( $2 \mathrm{~nm}$ thick) organic film (representing the adsorbed molecular layer) is used. Here we observe that, when a thin film is considered instead of the bulk refractive index, properly designed localized plasmon resonance sensors exhibit greater sensitivity compared to a prism coupler. Previously, various plasmonic resonators designed or fabricated on planar surfaces have been 
(a)

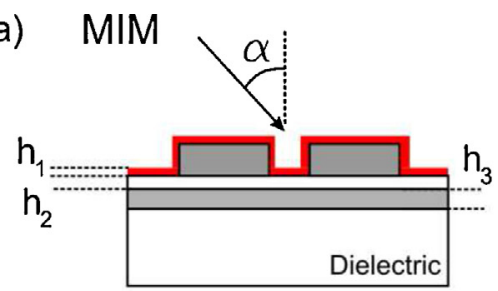

(b)

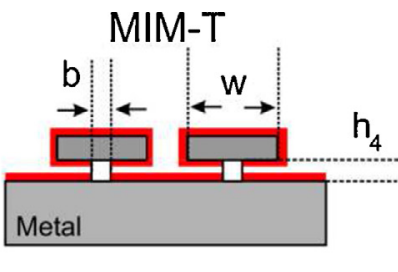

(c)

MMM-T

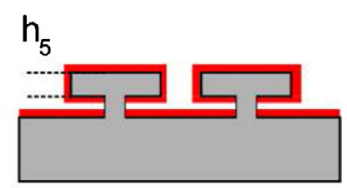

(d)

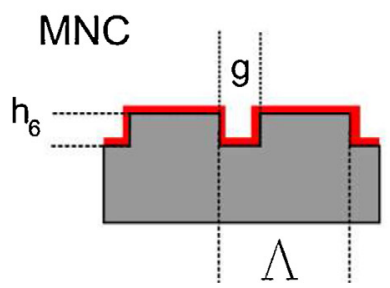

(e) $\mathrm{MI}-\mathrm{NI}$

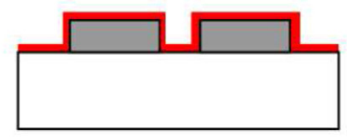

(f) Kretschmann

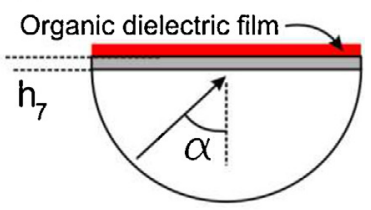

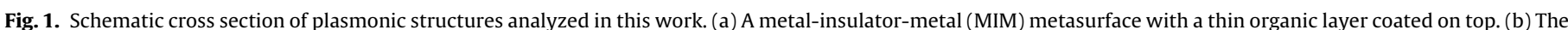

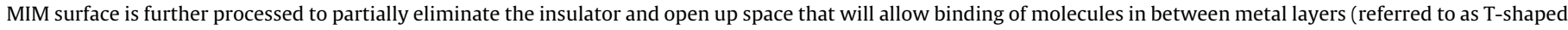

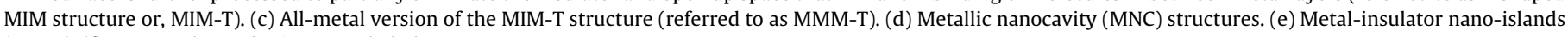
(MI-NI). (f) Commonly used prism-coupled plasmon resonance geometry.

considered as plasmonic interfaces for LSPR sensing $[15,16]$. We also consider plasmonic structures on planar surfaces that can be fabricated by top-down methods. We choose geometric structures that are straightforward to implement using standard nanofabrication techniques such as electron beam lithography. Particularly, we investigate the structures shown in Fig. 1. The structures include a metal-insulator-metal (MIM) metasurface with a thin organic layer coated on top (Fig. 1a). The MIM surface is further processed to partially eliminate the insulator and open up space that will allow binding of molecules in between metal layers (referred to as Tshaped MIM structure or, MIM-T, Fig. 1b). An all metal version of the MIM-T structure, referred to as MMM-T is shown in Fig. 1c. In addition to MIM structures, a metallic nanocavity (MNC) array is studied (Fig. 1d). The MNC is similar to a lamellar grating, however the rectangular grooves are geometrically designed to feature broad localized plasmon modes confined primarily into the groove, therefore serving as plasmonic nanocavities. The MNC layer structure allows fabrication by a simple nanoimprinting process. Also a metal-insulator nano-island (MI-NI) array is considered (Fig. 1e). Such structures can be fabricated by top-down fabrication techniques such as electron beam lithography or the geometry can be used to approximate self-organized metal nanoislands, typically formed by dewetting of thin metal films on glass substrates [21]. We compare the surfaces with the commonly used prism-coupled plasmon resonance geometry (Fig. 1f).

\section{Sensing by wavelength interrogation of plasmon resonance}

The propagation constant $k_{S P P}$ of the surface plasma wave propagating at the interface between a semi-infinite dielectric medium and a metal is given by [1]

$k_{s p p}=\frac{\omega}{c} \sqrt{\frac{\varepsilon_{m} \varepsilon_{d o}}{\varepsilon_{m}+\varepsilon_{d o}} \geq k_{0}=\frac{\omega}{c} \sqrt{\varepsilon_{d o}}}$

where $\varepsilon_{d}=n_{d o}^{2}$ and $\varepsilon_{m}=n_{m}^{2}$ are the dielectric functions of the dielectric and the metal, $\omega$ is the frequency and $k_{0}=2 \pi / \lambda_{0}$ is the free space wave vector. Since $k_{S P P}$ is larger than $k_{0}$, free space resonant coupling to such a mode is not possible and a prism of refractive index $n_{p}>1$ is generally used to couple free space light into the SPP mode. Resonant coupling takes place at an angle of incidence $\theta$, when

$k_{S P P} \cong n_{p} k_{o} \sin (\theta)$

In the prism coupled scheme (Kretschmann geometry), a thin dielectric film, of refractive index $n_{d}$ and thickness $h$, shifts the resonance according to [1]

$\delta k_{S P P} \cong \frac{K_{S P P}^{3}}{K_{0}^{2} n_{d o}^{3}}\left[1-\exp \left(-2 \gamma_{d} h\right)\right] \delta n_{d}$

where $\quad \gamma_{d}=i k_{o} \varepsilon_{d o} /\left(\sqrt{\left(\varepsilon_{m}+\varepsilon_{d o}\right)}\right) \quad$ and $h \ll 1 / \operatorname{Re}\left\{\gamma_{d}\right\} \quad$ and $\delta n_{d}=n_{d}-n_{d o}, n_{d o}$ being the bulk refractive index of the dielectric medium. In LSPR sensing using an isolated plasmonic resonator where the mode field can be assumed to isotropically decay into the surrounding medium, the wavelength shift upon binding is given similarly by $\delta \lambda=S_{\lambda} \delta n_{d}\left(1-\exp \left(-2 h / 1_{d}\right)\right)$, where $s \lambda$ is the sensitivity factor (shift in resonance per RIU change in environment refractive index) and $l_{d}$ is electromagnetic decay length of the LSPR mode [8-17]. However, for anisotropic resonators, such as prism or disk shaped, the mode field is non-uniformly distributed on the resonator and sensitivity of binding assumes a location dependent form. The same is true for resonators bound to a substrate, where only one side of the resonator is available for molecular binding. For a general plasmonic resonator including coupled resonators and cavities in buffer or on a surface, a simple equation including a single decay length cannot be used to express the wavelength shift. Instead, perturbation theory can be used to estimate the resonance wavelength shift upon a change in the refractive index profile through [18]

$\frac{\delta \lambda_{\text {res }}}{\lambda_{\text {res }}}=-\frac{1}{2} \frac{\int|E(r)|^{2}\left(n_{d}^{2}(r)-n_{d o}^{2}\right) d v}{\int|E(r)|^{2} d v}$

where $E(r)$ is the electric field distribution (mode profile), $n_{d o}$ is the refractive index profile before perturbation, $n_{d}(r)$ is the refractive index profile after the perturbation and integration is performed over all space.

Sensitivity for wavelength interrogated detection is [6]

$S_{\lambda}=\frac{\delta \lambda_{\text {res }}}{\delta n_{d}}$ 
The figure of merit (FOM) is defined as [9]

$\mathrm{FOM}=\frac{S_{\lambda}}{\Delta \lambda_{\text {res }}}=\frac{\delta \lambda_{\text {res }}}{\Delta \lambda_{\text {res }} \delta n_{d}}$

where $\Delta \lambda_{\text {res }}$ is the full-width-at-half-maximum (FWHM) of the resonance, $\delta \lambda_{\text {res }}$ is the wavelength shift, and $\delta n_{d}$ is the refractive index change in the dielectric film or bulk.

\section{Numerical calculation of mode profiles and refractive index sensitivity}

We use numerical computation (rigorously coupled wave analysis, RCWA) to calculate the spectral response of LSPR surfaces shown in Fig. 1 [19]. Previously, lumped circuit element models and transmission lines were used to calculate band structure for such coupled resonators [12]. Because of the complexity of the band structures of coupled LSPR resonators, we do not attempt to model the band structures using such models. Instead, the dimensions of the structures are optimized by RCWA calculations so that the band structures of the metasurfaces feature a resonance around 600-700 nm wavelength for normal incidence. Such a choice allows resistive losses to be similar, resulting in structures with similar resonance FWHM. We also choose dimensions that exhibit high sensitivity to refractive index change while keeping the structures large so that fabrication is more practical using top-down techniques. Structure sizes are $h_{1}=2 \mathrm{~nm}$ (film), $h_{2}=10 \mathrm{~nm}, h_{3}=50 \mathrm{~nm}$, grating height $h_{5}=50 \mathrm{~nm}$, period of $\Lambda=200$, gap of $g=35$ for the MIM (see Fig. 1 for dimension labels). For the MIM-T and MMM$\mathrm{T}, b=62.5 \mathrm{~nm}, w=200 \mathrm{~nm}$, top metal $h_{5}=10 \mathrm{~nm}, h_{4}=10 \mathrm{~nm}$, period $\Lambda=250 \mathrm{~nm}$, gap $g=50 \mathrm{~nm}$. For MNC, $h_{6}=40 \mathrm{~nm}$, period $\Lambda=200 \mathrm{~nm}$, gap $g=35 \mathrm{~nm}$. For MI-NI, top metal thickness $h_{5}=100 \mathrm{~nm}$, period $\Lambda=200 \mathrm{~nm}$, gap $g=25 \mathrm{~nm}$ and for the Kretschmann configuration $h_{7}=50 \mathrm{~nm}$.

The electric field distributions of the structures excited on resonance at normal incidence are shown in Fig. 2. For the MIM structure, on resonance the fields are primarily confined to the insulator and the space between top metal islands (Fig. 2a). According to Eq. (4), MIM structures are expected to have a lower sensitivity to changes of the refractive index of the background, since fields are partially confined to the dielectric inside the MIM. In contrast, the MIM-T structures have a larger surface to where the molecules can be bound (Fig. 2b). The MMM-T configuration displays fields that are similar to the MIM-T, however, field penetration into the intermediate metal post region is smaller and greater fraction of the field is confined to the exposed areas (Fig. 2c). The MNC structure has all the fields confined within the nanocavity, which is a completely exposed surface (Fig. 2d). The MI-NI structures are similar to the MNC, however field penetration into the dielectric reduces the fraction of mode within the exposed areas (Fig. 2e). In the Kretschmann configuration, the SPP mode is confined to the surface, however the mode volume is large due to the large decay length into the dielectric (Fig. 2f).

The absorption spectra of the structures are calculated by RCWA and are shown in Fig. 3. The LSPR structures may possess further resonances outside the wavelength range shown, however we are only concerned about the resonance near $650-850 \mathrm{~nm}$. The absorption is calculated for normal incidence for changes in refractive index of a $2 \mathrm{~nm}$ thick film on a $n=1.33$ background. As can be seen in Fig. 3, an increase in the refractive index of the film typically results in a red shift of the resonance lines due to dielectric loading of the resonators.

The fields calculated by numerical calculations are used for estimation of the resonance shift through Eq. (5) and are given in Table 1 . The results are acquired for resonance excitation and for a shift of the background index. The shifts are compared with direct calculation of resonance frequencies using RCWA and Eq. (5) (Eq.
Table 1

Fractional shift of resonance wavelength upon \%1 change of background refractive index, around $n=1.33$, calculated using Eq. (5) and direct finite element analysis.

\begin{tabular}{|c|c|c|}
\hline Structure & Eq. (5) & Direct FEM spectra \\
\hline MIM & 0.00088 & 0.00095 \\
\hline MIM-T & 0.00430 & 0.00410 \\
\hline MMM-T & 0.00470 & 0.00490 \\
\hline MI-NI & 0.00350 & 0.00390 \\
\hline MNC & 0.00450 & 0.00600 \\
\hline Prism $^{\mathrm{a}}$ & 0.06130 & 0.070000 \\
\hline
\end{tabular}

(3) for the prism) are observed to agree within $25 \%$ with numerical calculations. The agreement can be considered reasonable and the discrepancy is attributed to the finite mesh size used in numerical calculations. The results show the validity of the perturbation approach. Therefore, intuitively we can expect the structures with greater exposed area and smaller mode volume to exhibit improved sensitivity to the presence of thin molecular films. It must be noted that, in order to achieve correct results using Eq. (5), the dielectric function of the metal film and fields penetrating inside the metal structures must be included in the calculation. Otherwise, for example by incorrectly assuming zero field penetration into the metal and assuming the dielectric portions of the resonator account for all of the mode volume, the perturbation approach produces erroneous resonance shifts that are 100-300\% larger than those predicted by direct numerical calculation. Therefore, mode energy inside the metal surfaces contributes a significant portion of the actual mode volume.

An organic molecular film is expected to have a refractive index of 1.47 around $600-800 \mathrm{~nm}$ and the index contrast with buffer solution $\left(n_{d}=1.33-1.34\right)$ results in the perturbation of resonances. We calculate the absorption spectra of surfaces using RCWA as shown in Fig. 3, and extract peak positions of the absorption peaks around $650-800 \mathrm{~nm}$ to calculate sensitivity to the refractive index of a $2 \mathrm{~nm}$ thick film as well as to the background refractive index (Table 2). It is observed that, Kretschmann configuration surpasses all LSPR structures in sensitivity if the background refractive index is changed. However, for a thin organic film, due to the small mode volume of the LSPR resonators, several LSPR structures show improved response over the prism. For example, a comparison of the spectral shifts of MIM-T and Kretschmann configurations is given in Fig. 4 for thin film and background refractive index change. In the presence of a thin film, a larger shift of resonance frequency of the LSPR structure compared to a prism is interesting (Fig. 4a and b), since prism coupling is typically accepted to be superior to other SPP structures (such as grating coupled plasmon resonance [6]) in terms of wavelength sensitivity. A thin organic film is also of greater practical interest than bulk background refractive index change, as this configuration more accurately describes an actual molecular binding experiment. For the thin film case, it is seen that all LSPR structures except the MIM exhibit better absolute sensitivity compared to the prism. The MIM is an exception, being less sensitive due to the fact that greater portion of the mode is

Table 2

Wavelength sensitivity upon $\% 1$ change of refractive index of $2 \mathrm{~nm}$ thin film, around $n=1.47$, compared with $\% 1$ change of background index near $n=1.33$, calculated using finite element analysis.

\begin{tabular}{llc}
\hline Structure & $2 \mathrm{~nm}$ film $(\mathrm{nm} / \mathrm{RIU})$ & Background $(\mathrm{nm} / \mathrm{RIU})$ \\
\hline MIM & 18.9 & 60 \\
MIM-T & 97.3 & 330 \\
MMM-T & 78.4 & 330 \\
MI-NI & 59.5 & 260 \\
MNC & 56.8 & 390 \\
Prism & 48.7 & 4500 \\
\hline
\end{tabular}



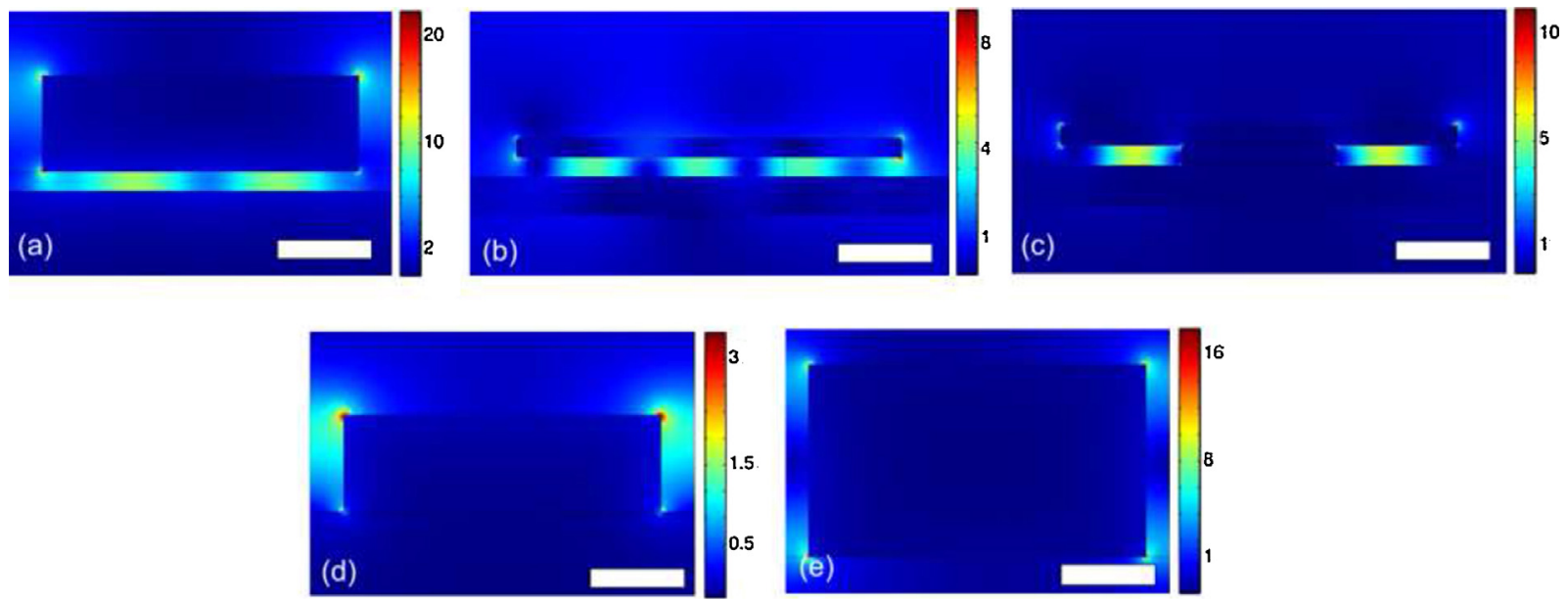

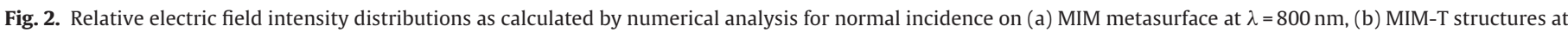
$\lambda=750 \mathrm{~nm}$, (c) MMM-T structures at $\lambda=625 \mathrm{~nm}$, (d) MNC surface at $\lambda=650 \mathrm{~nm}$, (e) MI-NI structures at $\lambda=650 \mathrm{~nm}$. Scale bars are $50 \mathrm{~nm}$ wide.

confined in the unexposed insulator region. The MIM-T and MMM$\mathrm{T}$ feature about twice the sensitivity of a prism, partially due to the fact that both top and bottom surfaces have $2 \mathrm{~nm}$ film covering them and there is about twice more material per unit area that contribute to resonance wavelength shift. If we calculate the FOM given by Eq. (6), for a $2 \mathrm{~nm}$ film, we get values of 0.34 for the MIM, 0.62 for the MI-NI, 0.44 for the MNC, 1.35 the MIM-T, 0.91 for the MMM-T and 0.87 for the Kretschmann. In terms of the FOM, the MIM-T and MMM-T exhibit improved performance compared to the prism coupled SPR. It must be noted that, for LSPR sensing by resonators in fluid, a refractive index sensitivity of $\sim 500 \mathrm{~nm} / \mathrm{RIU}$ is considered desirable [20] It is observed that for the structures except the MIM, sensitivities of 300-400 nm/RIU can be achieved with surface bound structures considered here. The sensitivity for LSPR structures on surfaces is inherently low as compared to free structures in fluid, due to single sided molecular binding. For surface bound structures, sensitivity values ranging from $80 \mathrm{~nm} / \mathrm{RIU}$ have been previously reported [16]. The sensitivity can be increased to $750 \mathrm{~nm} /$ RIU for vertical cavity structures similar to the MIM-T, designed in the near-infrared portion of the spectrum. This increase was at the expense of increased fabrication tolerances and a potentially complicated fabrication procedure [15].

Another issue we address in comparing the performance of the LSPR sensors is the angular sensitivity of the resonances. In the case of a focused beam with a half-cone angle $\Delta \theta$, the broadening in the wavelength response of a plasmonic resonance will be given to a first order approximation by $\Delta \lambda_{\theta} \cong 2\left(\partial \lambda_{\text {res }} / \partial \theta\right) \Delta \theta$. The resonance will feature a peak given by the convolution of the angular spread with the intrinsic resonance lineshape, resulting in an overall resonance width of $\Delta \lambda_{\text {total }} \cong \sqrt{\Delta \lambda_{\text {res }}^{2}+\Delta \lambda_{\theta}^{2}}$. Rewriting the angular
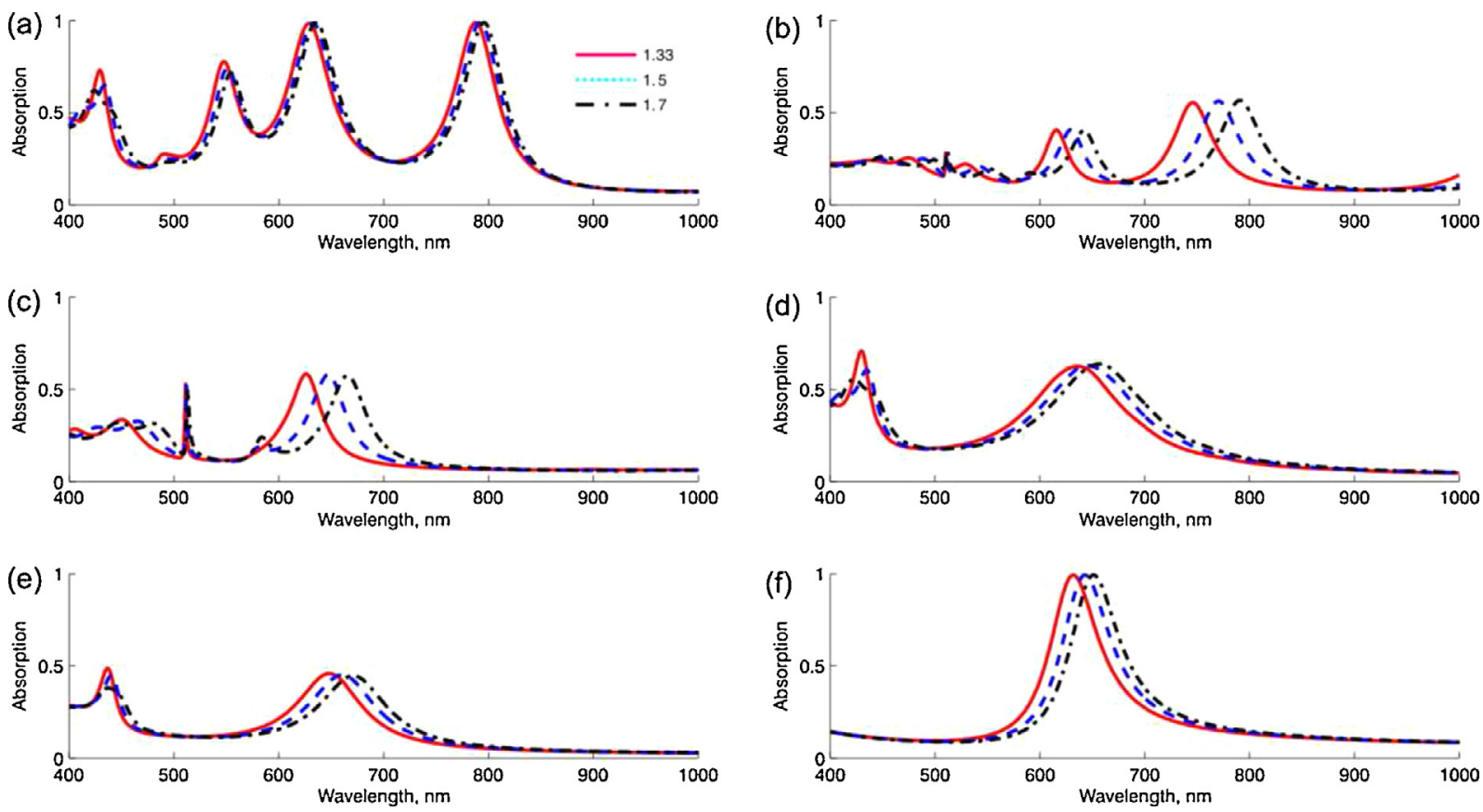

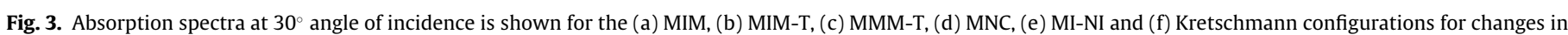
the refractive index of a $2 \mathrm{~nm}$ thick film with $n=1.33,1.5$ and 1.7. A background refractive index of $n_{d}=1.33$ is used in the calculations. 

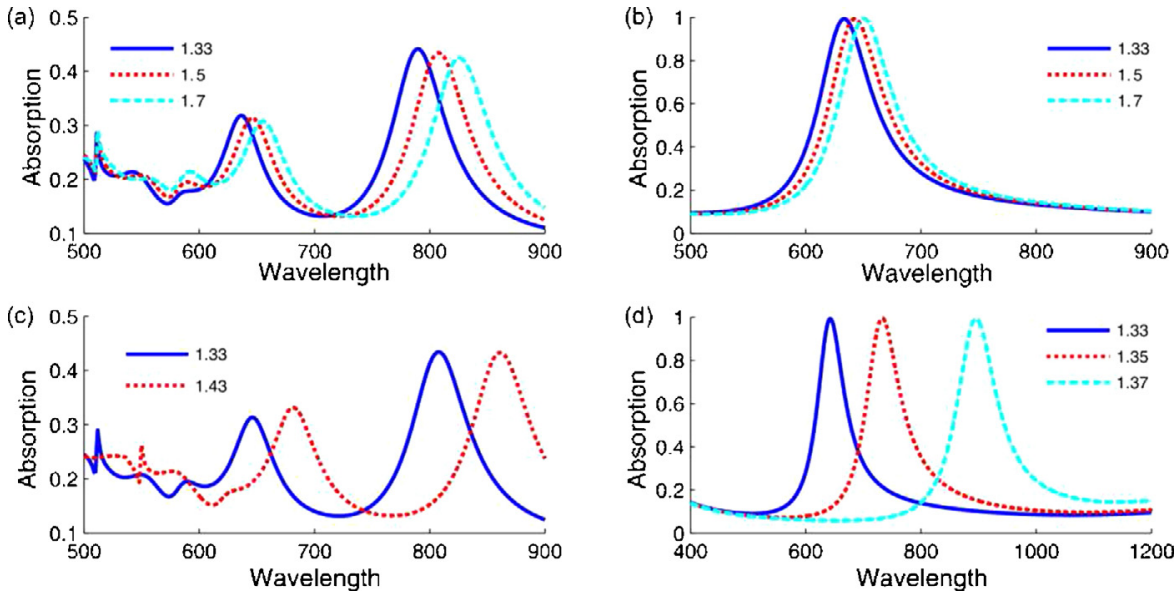

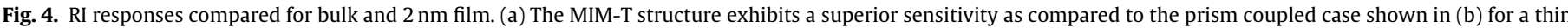

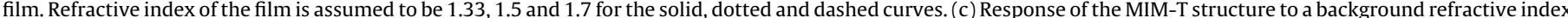

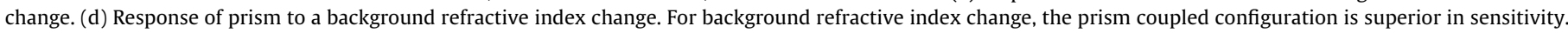
Legend shows refractive indices of the background for each graph.

(a)

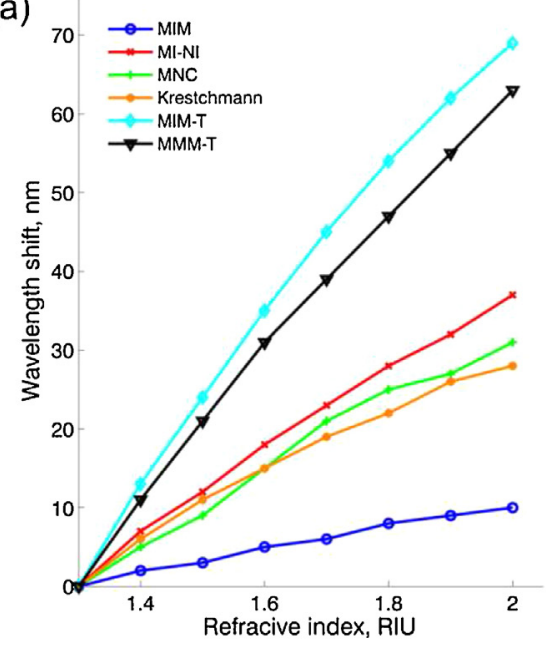

(b)

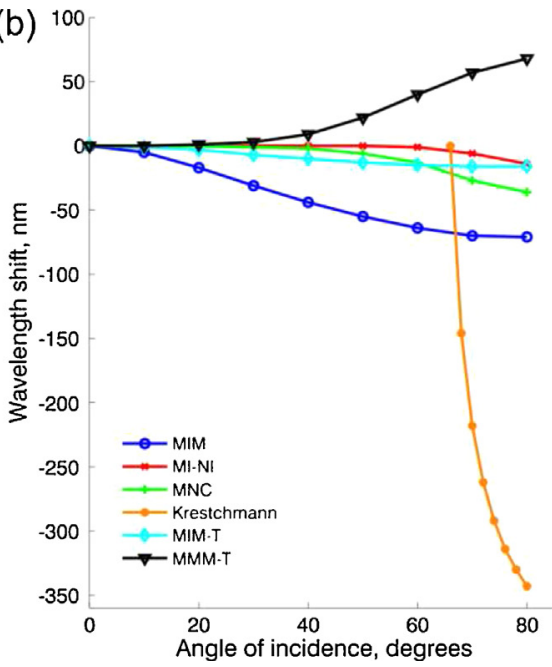

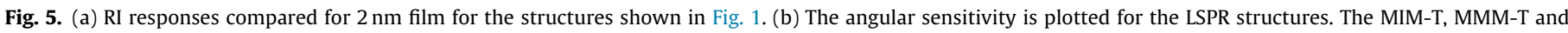

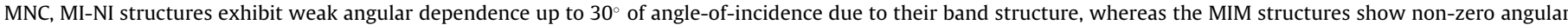
sensitivity. The Kretschmann configuration has inherently a very high angular sensitivity.

spread in terms of the numerical aperture of the focusing lens (N.A.), for small N.A. the figure of merit will be given approximately by

$F O M \cong F O M_{0} \frac{\Delta \lambda_{\text {res }}}{\sqrt{\Delta \lambda_{\text {res }}^{2}+\left[2\left(\partial \lambda_{\text {res }} / \partial \theta\right)(\text { N.A. } / n)\right.}}$

where $F O M_{O}$ is the figure of merit measured using a collimated beam. In the case of an omni-directional resonance, $\partial \lambda_{\text {res }} / \partial \theta \approx 0$ and a large N.A. would not degrade the FOM, allowing high spatial resolution imaging. The angular sensitivity is typically large for a prism coupled configuration, however LSPR surfaces typically exhibit angle independent spectral response. Angular responses of the LSPR resonance are shown in Fig. 5b, along with spectral response to $2 \mathrm{~nm}$ thick films of varying refractive index (Fig. 5a). It is seen that, for the MIM-T, MMM-T and MI-NI surfaces, the $\Delta \theta \partial \lambda_{\text {res }} / \partial \theta \ll \Delta \lambda_{\text {res }}\left(\right.$ i.e. $\partial \lambda_{\text {res }} / \partial \theta \approx 0$ ) is satisfied for angles up to $30^{\circ}$. This would allow a N.A. $=0.66$ to be used (in buffer with $n=1.33$ ) for colorimetric imaging of LSPR resonances, resulting in a diffraction limited resolution of $490 \mathrm{~nm}$ assuming a resonance with peak around $650 \mathrm{~nm}$. The actual band structure of coupled LSPR structures determine the value of angular sensitivity $\lambda_{\text {res }} / \partial \theta$ and MIM-T, MMM-T and MI-NI surfaces have superior omni-directionality as compared to the MIM surface for the set of geometric parameters given in Table 1.

\section{Conclusion}

We have compared the refractive index response of several LSPR structures and prism coupled SPR in the presence of a thin organic film. It is seen that for a bulk background refractive index change, the prism possesses superior refractive index sensitivity while LSPR structures can exhibit improved sensitivity for a $2 \mathrm{~nm}$ thick film. The main reason behind this improved sensitivity is the fact that the modes are confined to small volumes with $10-30 \mathrm{~nm}$ characteristic dimensions for the LSPR case, where a greater fraction of the mode is perturbed by the presence of the thin film. It is also shown that, if the LSPR modes are confined to regions where biomolecular adhesion is inhibited by the presence of a dielectric, as in the MIM case, sensitivity is degraded, since a smaller portion of the mode volume is perturbed by biomolecular adhesion. Calculations validate the applicability of the perturbation approach to calculating the refractive index response, as seen by comparing Eq. (5) with direct numerical calculations. In the calculations, it was 
seen that geometric volume of the metal-free region of the resonators does not correspond to the actual mode volume, which needs to be calculated taking into account the metal dielectric constants and fields confined inside the metal regions. Therefore, although the perturbation approach provides intuition, the fields that penetrate into the metal layer must be considered, as the metal dielectric function may have a large magnitude as compared to organic layers. The angular responses of the LSPR structures are also calculated, demonstrating the relative insensitivity of LSPR structures to changes in the angle of incidence. The results show that, properly designed LSPR structures retain omni-directionality while maintaining a higher sensitivity as compared to a prism for a thin organic film. Particularly, the MIM-T type resonators exhibited about 2 times improved sensitivity over a prism. Therefore, the calculations show that high resolution colorimetric plasmon resonance imaging is possible using optimized LSPR surfaces without compromising sensitivity for biomolecular detection.

\section{Acknowledgment}

This work was partially supported by TUBITAK under Grant 111M344, EU FP7:People-IAPP NanoBacterPhageSERS.

\section{References}

[1] J. Homola (Ed.), Surface Plasmon Resonance Based Sensors, Springer Series on Chemical Sensors and Biosensors, Vol. 4, Springer-Verlag, 2006.

[2] J. Homola, Surface plasmon resonance sensors for detection of chemical and biological species, Chem. Rev. 108 (2008) 462-493.

[3] B. Liedberg, I. Lundstrom, E. Stenberg, Principles of biosensing with an extended coupling matrix and surface plasmon resonance, Sens. Actuators B 11 (1993) $63-72$.

[4] L.M. Zhang, D. Uttamchandani, Optical chemical sensing employing surface plasmon resonance, Electron. Lett. 23 (1988).

[5] Ch. Striebel, A. Brecht, G. Gauglitz, Characterization of biomembranes by spectral elipsometry, surface plasmon resonance and interferometry with regard to biosensor application, Biosens. Bioelectron. 9 (1994) 139-146.

[6] H. Jiri, K. Ivo, S.S. Yee, Surface plasmon resonance sensors based on diffraction gratings and prism couplers: sensitivity comparison, Sens. Actuators B 54 (1999) 16-24.

[7] M. Stewart, C. Anderton, L. Thompson, J. Maria, S. Gray, et al., Nanostructured plasmonic sensors, Chem. Rev. 108 (2) (2008) 494-521.

[8] K.A.R. Willets, P. Van Duyne, Localized surface plasmon resonance spectroscopy and sensing, Ann. Rev. Phys. Chem. 58 (1) (2007) 267-297.

[9] A.A. Yanik, A.E. Cetin, M. Huang, A. Artar, S. Hossein Mousavi, A. Khanikaev, J.H. Connor, G. Shvets, H. Altug, Seeing protein monolayers with naked eye through plasmonic Fano resonances, Proc. Natl. Acad. Sci. 108 (29)(2011) 11784-11789.

[10] T.G. Terry, L. Hye Jin, M. Robert, CornDirect detection of genomic DNA by enzymatically amplified SPR imaging measurements of RNA microarrays, J. Am. Chem. Soc. 126 (2004) 4086-4087.
[11] J. Bianca Beusink, A.M.C. Lokate, G.A.J. Besselink, G.J.M. Pruijn, R.B.M. Schasfoort, Angle-scanning SPR imaging for detection of biomolecular interactions on microarrays, Biosens. Bioelectron. 23 (2008) 839-844.

[12] S. Ayas, H. Güner, B. Türker, O.Ö Ekiz, F. Dirisaglik, A.K. Okyay, A. Dâna, Raman enhancement on a broadband meta-surface, ACS Nano 6 (8) (2012) 6852-6861.

[13] M. Najiminaini, F. Vasefi, B. Kaminska, J.L. Jeffrey, Carson effect of surface plasmon energy matching on the sensing capability of metallic nano-hole arrays, Appl. Phys. Lett. 100 (2012) 063110.

[14] T.-Y. Chang, M. Huang, A.A. Yanik, H.-Y. Tsai, P. Shi, S. Aksu, M.F. Yanik, H. Altug, Large-scale plasmonic microarrays for label-free high-throughput screening, Lab Chip 11 (2011) 3596.

[15] O. Saison-Francioso, G. Lévêque, A. Akjouj, Y. Pennec, B. Djafari-Rouhani, R. Boukherroub, S. Szunerits, Search of extremely sensitive nearinfrared plasmonic interfaces: a theoretical study, Plasmonics (2013) 1-8, http://dx.doi.org/10.1007/s11468-013-9588-9.

[16] E. Galopin, et al., Sensitivity of plasmonic nanostructures coated with thin oxide films for refractive index sensing: experimental and theoretical investigations, J. Phys. Chem. C 114 (27) (2010) 11769-11775.

[17] S. Szunerits, R. Boukherroub, Sensing using localised surface plasmon resonance sensors, Chem. Commun. 48 (72)(2012) 8999-9010.

[18] J.D. Joannopoulos, S.G. Johnson, J.N. Winn, R.D. Meade, Photonic Crystals: Molding the Flow of Light, 2nd ed, Princeton Univ. Press, Princeton, 2008.

[19] M.G. Moharam, T.K. Gaylord, Rigorous coupled-wave analysis of metallic surface-relief gratings, J. Opt. Soc. Am. A 3 (1986) 1780.

[20] J.N. Anker, et al., Biosensing with plasmonic nanosensors, Nat. Mater. 7 (6) (2008) 442-453.

[21] S. Ayas, G. Cinar, A.D. Ozkan, Z. Soran, O.O. Ekiz, D. Kocaay, A. Tomak, P. Toren, Y. Kaya, I. Tunc, H. Zareie, T. Tekinay, A.B. Tekinay, M.O. Guler, A. Dana, Label-Free Nanometer-Resolution Imaging of Biological Architectures through Surface Enhanced Raman Scattering, Scientific Reports 3 (2013) 2624.

\section{Biographies}

Yasin Kaya is a research assistant at the UNAM Materials Science and Nanotechnology Program at Bilkent University. His research interests are in the field of computational electromagnetics and design of plasmonic nanostructures.

Sencer Ayas is a Ph.D. student at the UNAM Materials Science and Nanotechnology Program at Bilkent University. His research is on the design and realization of plasmonic nanostructures and metasurfaces, with applications to imaging and spectroscopy.

Ahmet Emin Topal is a Ph.D. student at the UNAM Materials Science and Nanotechnology Program at Bilkent University. His research interests are in the field of colorimetric biomolecular sensing and imaging using plasmon resonances.

Hasan Güner is a Ph.D. student at the UNAM Materials Science and Nanotechnology Program at Bilkent University. His research interests are in the field of design and implementation of plasmon resonance sensing systems.

Aykutlu Dana is a Associate Professor at the UNAM Materials Science and Nanotechnology Program at Bilkent University. He received his Ph.D. from Stanford University and continues to work in the fields of nanoscale optoelectronic devices and sensors. 